\title{
ADEQUATE UV EXPOSURES FOR HEALTHY LIFE: IN SITU MONITORING AND MODEL CALCULATION OF THE VITAMIN-D-SYNTHETIC CAPACITY OF SUNLIGHT
}

\author{
Irina Terenetskaya, Tetiana Orlova, Pavel Kapinos \\ Institute of Physics, National Academy of Sciences of Ukraine \\ 03028, Prospekt Nauki, 46, Kiev-28, Ukraine, \\ Tel: +38044525 0813,Fax: +380445251589, e-mail:teren@iop.kiev.ua
}

\begin{abstract}
Vitamin D which is formed upon UV solar radiation in human skin is essential in many physiological functions. To estimate beneficial vitamin-D-synthetic capacity of sunlight a bio-equivalent UV dosimeter that is based on the same molecular photochemistry from which vitamin D is photosynthesized in human skin has been developed. The examples of an in situ monitoring of the vitamin-D-synthetic capacity of sunlight using an in vitro model of vitamin D synthesis are presented, and various operational principles of the UV biodosimeter are discussed. In addition, reliable algorithm is presented for direct calculation of previtamin $\mathrm{D}_{3}$ accumulation using the photoreaction mathematical model with solar UV spectra as input data. Critical dependence of previtamin $\mathrm{D}_{3}$ accumulation on cloudiness and aerosols is demonstrated.
\end{abstract}

Keywords: Solar UV radiation, vitamin D synthesis, UV monitoring, UV biodosimeter, mathematical model.

\section{Introduction}

It is well understood now that the appropriate doses of UV sunlight are beneficial for humans initiating vitamin $\mathrm{D}^{1^{*}}$ synthesis in skin as opposed to excessive UV exposures that are generally associated with acute and chronic health effects (erythema, skin cancer, immune system suppression, cataract, etc.). Natural exposure to sunlight is responsible for maintaining adequate vitamin D nutrition for most of the population in the world, and the importance of sunshine as a therapeutic measure for preventing the bone deforming diseases is now appreciated. Moreover, in addition to the well-established effects of vitamin D on maintenance of mineral homeostasis, recent evidences suggest a wider biologic role of vitamin D not primarily related to mineral metabolism [2-4]. The findings of recent epidemiological studies [5] demonstrate that vitamin D can significantly reduce the risk for several types of cancer (breast, colon, kidney, myeloid leukemia, ovary, pancreas, prostate), and the cancer mortality rates are correlated inversely with local solar UV-B doses. The most likely mechanism whereby solar UV-B radiation provides protection against cancer is natural production of vitamin D.

Sunlight has long been recognized as the main source of vitamin $\mathrm{D}_{3}$ for humans, and all higher vertebrates as well as most plants, zooplankton, avian species and aquatic mammals have an endogenous mechanism involving solar UV radiation that affects the synthesis of vitamin D from its precursor [6].

These facts testify that an in situ control of solar UV radiation with respect to its ability to initiate vitamin D synthesis demands particular care, especially in view of dramatic effect of seasonal and latitudinal changes of the UV-B intensity on the vitamin D synthetic capacity of sunlight [7]. (There are numerous observations that people previously living near equator have severe vitamin D deficiency and the associated health problems when moving to the North). It is just sharp decrease in provitamin D absorbance spectrum from its maximum at $282 \mathrm{~nm}$ to the longer wavelengths that implies selective sensitivity of vitamin D synthesis solely to the UV-B part $(280-315 \mathrm{~nm})$ of solar spectrum which is very changeable (Figure 1). The solar zenith angle change (daily, seasonal and latitudinal) is the governing factor, but clouds, aerosols, albedo and air pollution also affect the UV-B irradiance at the Earth surface. Just adverse effects of air pollution include primary health effects, environmental acidification, and possible long-term changes in the global climate and in UV radiation levels. As one can see from Figure 1, the absorption spectrum of $\mathrm{SO}_{2}$ overlaps UV-B region and therefore increase in its level due to volcanic eruptions can inhibit the vitamin D synthesis.

\footnotetext{
1 * Vitamin D is represented by cholecalciferol (vitamin D3) and ergocalciferol (vitamin D2) that are structurally similar secosteroids derived from the UV irradiation of provitamin D sterols. (Secosteroids are steroids in which one of the rings has broken). In vertebrates vitamin D3 is produced in vivo by the action of sunlight on 7-dehydrocholesterol (7-DHC) in the skin. Vitamin D2 is produced in plants, fungi and yeasts by the solar irradiation of ergosterol.

Vitamin D2 and vitamin D3 differ structurally only in the C-17 side-chain, which in vitamin D2 has a double-bond and an additional methyl group. Irradiation of parent steroid results in breakage of the B-ring at the 9,10-carbon bond, resulting in the conjugated triene system of double bonds in. previtamin D molecule [1].
} 


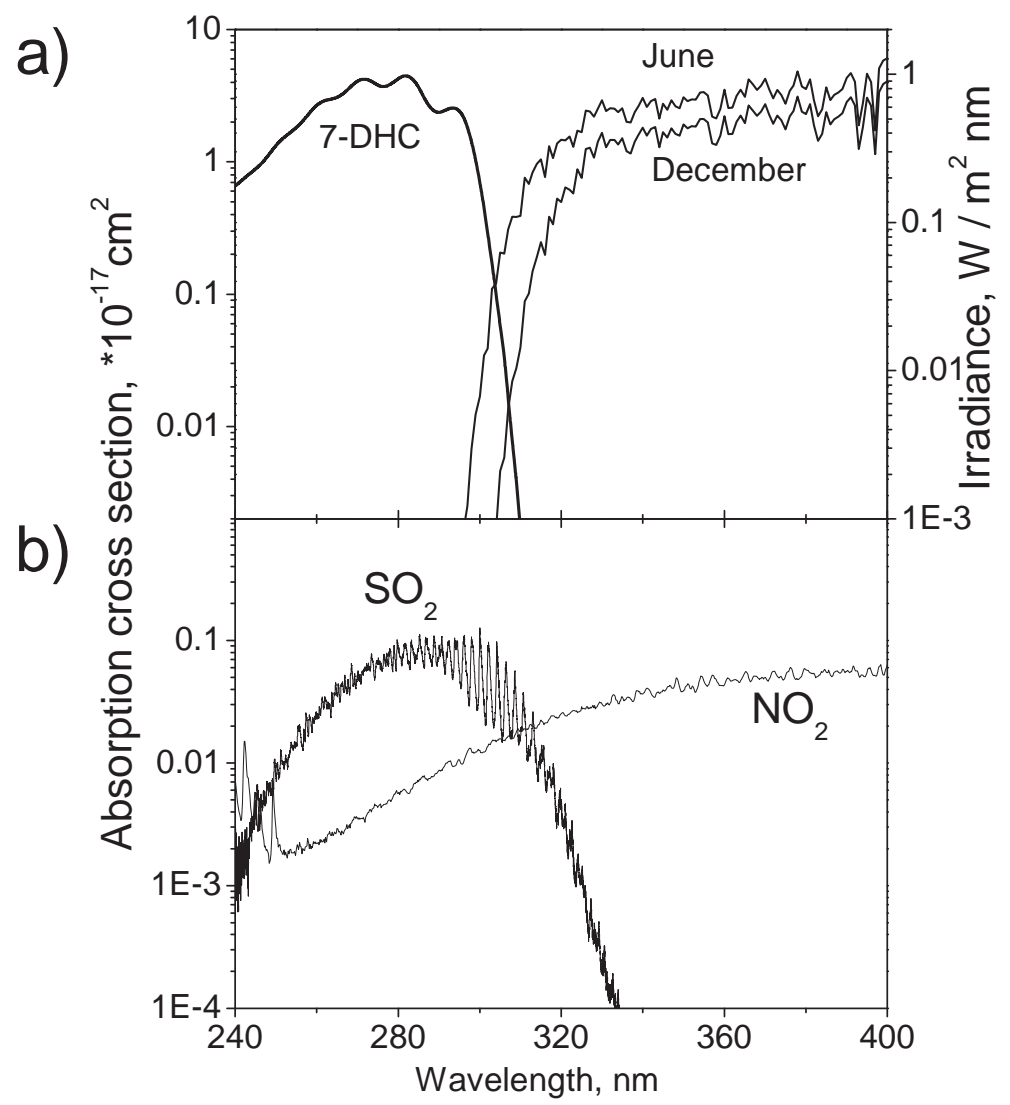

Figure 1. Absorption cross section of 7-DHC (provitamin D3) molecule versus solar UV spectra calculated by FASTRT program [8] (http://nadir.nilu.no/ olaeng/fastrt/) for Kiev (50 $\left.27^{\prime} \mathrm{N}, 30^{\circ} 31^{\prime} \mathrm{E}\right)$, June, 22 and December, 22, 10:00 GMT, total ozone column 300 DU, clouds- and aerosol-free sky (a), and absorption cross section of gaseous molecules $\mathrm{SO}_{2}$ and $\mathrm{NO}_{2}$ [9] (www.atmosphere.mpg.de/spectral-atlas-mainz) (b).

\section{Methodical part}

Prediction of biologic effects of solar radiation on human health, in particular, involving terrestrial level of solar UV radiation, requires development of adequate methodological strategy.

But as far as excessive UV exposures cause negative health effects, in most cases biological activity of solar UV radiation is calculated by weighting solar UV spectra with CIE erythema action spectrum [10]. Yet, the beneficial vitamin D synthetic capacity of sunlight cannot be correctly estimated by this way in view of significant difference between the erythemic and the vitamin $\mathrm{D}$ synthesis action spectra [11,12]. With due regard to the essential role of vitamin $\mathrm{D}_{3}$ for human health we have developed a bio-equivalent $\mathrm{UV}$ dosimeter that is based on the same molecular photochemistry from which vitamin $\mathrm{D}$ is photosynthesized in human skin, i.e. 7-dehydrocholesterol (provitamin $\mathrm{D}_{3}$ ) photoconversion [13-17].

The complex network of vitamin D synthesis consists in the two stages of monomolecular isomerizations [16]. At the first stage UV irradiation of provitamin D within its absorption band (240-315 nm) yields previtamin D, which further is converted into vitamin D (Figure 2) and just amount of previtamin D accumulated during an UV exposure is a measure of biologically active UV dose.

However, upon UV-B radiation previtamin D itself undergoes a number of side photoconversions. As a result, UV irradiation of initial provitamin $\mathrm{D}$ gives rise to formation of multicomponent photoisomer mixture which composition is highly dependent on the irradiation wavelength $[19,20]$.

1. Original spectrophotometric (SPM) analysis which takes into account irreversible photodegradation has been designed to follow the photoreaction course in real time as alternative to the time-consuming HPLC analysis [15]. This opened the way to an in situ UV monitoring using the photochemical stage of vitamin D synthesis (in vitro model). 


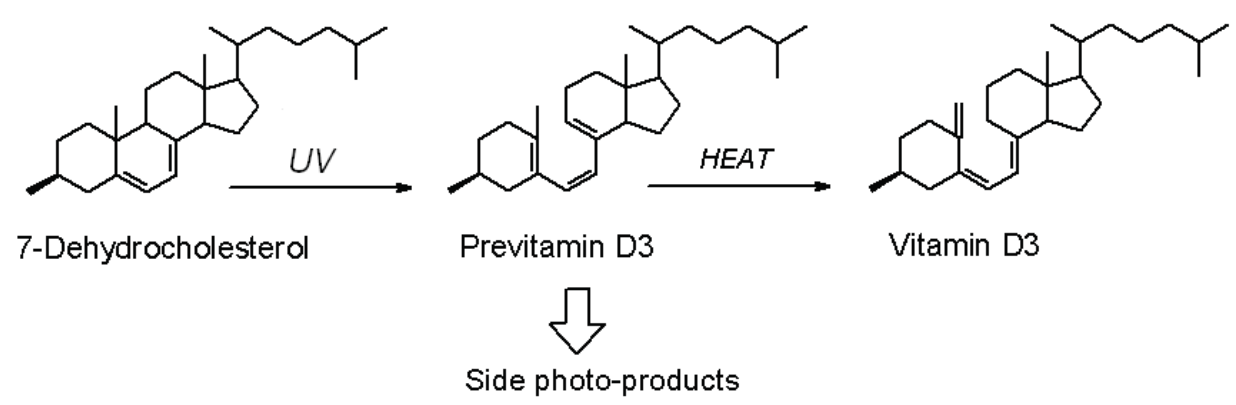

Figure 2. Simplified scheme of vitamin D3 synthesis from initial 7-Dehydrocholesterol.

With this aim solution of 7-dehydrocholesterol (provitamin $\mathrm{D}_{3}$ ) in ethanol is exposed to sunlight in rectangular quartz cuvette, and the solution absorption spectrum is recorded before and after an exposure with UV spectrophotometer. The previtamin D concentration (accumulated 'antirachitic' UV dose) is determined by computer processing of the recorded spectra using specially designed computer program which allows deriving the photoisomer concentrations based on the photoisomer individual absorption spectra [21]. An important point is that the ambiguity in the solution of the over determined system of equations requires expertise in pursuance of concentration analysis. Therefore, for general use, the simplified method of UV dosimetry was introduced [17] similar to a number of chemical UV dosimeters as, for instance, polysulphone film, that measured an accepted UV dose by the absorbance decrease at the fixed wavelength [22].

2. Previous data on transformation of provitamin $\mathrm{D}$ absorption spectrum during the summer day exposure to sunlight in Greece [14] suggest that the 'antirachitic' UV dosimetry can be carried out by recording the absorbance decrease at $\lambda=282 \mathrm{~nm}$ as a function of exposure time after a calibration procedure. As was shown in [17], the absorbance decrease plotted as a function of the previtamin $\mathrm{D}$ concentrations determined by the spectrophotometric analysis was described rather well by a linear approximation $Y=A+B x:(A=0,995, B=-0.0096, R=-0.996, S D=0.0069, \mathrm{R}=$

correlation coefficient, $\mathrm{SD}$ - standard deviation). Therefore the previtamin D concentration $\left(C_{\operatorname{Pr} e}(\%)\right.$ can be calculated from the absorbance $\boldsymbol{A}$ at $\lambda=282 \mathrm{~nm}$ before $\left(A_{282}^{0}\right)$ and after $\left(A_{282}^{t}\right)$ UV irradiation using the formula:

$$
C_{\mathrm{Pr} e}(\%)=\left(1-A_{282}^{t} / A_{282}^{0}\right) / 0.01
$$

Although comparison of calculated previtamin D concentrations during long term monitoring of 'antirachitic' solar UV radiation in Kiev $\left(50^{\circ} 23^{\prime} \mathrm{N}, 30^{\circ} 32^{\prime} \mathrm{E}\right)$ showed larger data scatter, nevertheless, the correlation coefficient $\mathrm{R}$ (between previtamin D concentrations determined by means of the optical density decrease at $282 \mathrm{~nm}$ and those ones determined by spectrophotometric analysis) was rather high $(R=0.77)$ within upper and lower $90 \%$ prediction limits [17].

3. These results provided a basis for the new method of in situ determination of a vitamin D synthesizing amount of natural and artificial UV irradiation [23]. The method comprises: (1) exposing a polymer film doped with 7-dehydrocholesterol to an UV radiation the vitamin-D synthesizing amount of which is to be determined; (2) measuring change of absorbance of the film before and after an exposition to the UV radiation using specially elaborated optoelectronic device (or UV spectrophotometer); and (3) correlating the measured change of the absorbance to a previtamin D synthesizing amount.

4. Finally, to visualize the process of previtamin $\mathrm{D}$ photosynthesis and provide the easiest detection of previtamin D synthesis the optically active 7-DHC (provitamin $\mathrm{D}_{3}$ ) molecules were dissolved in liquid crystalline (LC) matrix [24]. It was assumed that alteration of molecular geometry of provitamin $\mathrm{D}$ by the photoinduced conversion into previtamin $\mathrm{D}$ should affect the selective reflection and, as a result, the LC cell color [25, 26]. This could provide the easiest detection of previtamin D synthesis and evaluation of the accumulated UV dose in situ by comparison of the LC cell color with the calibration scale (like litmus paper measures a solution $\mathrm{pH}$ ).

5. Additionally, the vitamin D synthetic capacity of sunlight can be directly calculated using adequate mathematical model which enables calculation of the photoreaction kinetics for any UV radiation source with known spectral irradiance, and the results of the laboratory and field tests lent validity of both the model and the spectrophotometric analysis [14, 17].

The kinetics of provitamin D photoisomerization is described by the following system of rate equations (2) 


$$
\begin{aligned}
\frac{d C_{1}}{d t} & =\int d \lambda I^{*}(\lambda)\left[-\varepsilon_{1}(\lambda) C_{1} \phi_{12}+\varepsilon_{2}(\lambda) C_{2} \phi_{21}\right] \\
\frac{d C_{2}}{d t} & =\int d \lambda I^{*}(\lambda)\left[-\varepsilon_{2}(\lambda) C_{2} \phi_{21}+\varepsilon_{1}(\lambda) C_{1} \phi_{12}-\varepsilon_{2}(\lambda) C_{2} \phi_{23}+\varepsilon_{3}(\lambda) C_{3} \phi_{32}-\varepsilon_{2}(\lambda) C_{2} \phi_{24}+\varepsilon_{4}(\lambda) C_{4} \phi_{42}\right. \\
& -\varepsilon_{2}(\lambda) C_{2} \phi_{25} \\
\frac{d C_{3}}{d t} & =\int d \lambda I^{*}(\lambda)\left[-\varepsilon_{3}(\lambda) C_{3} \phi_{32}+\varepsilon_{2}(\lambda) C_{2} \phi_{23}\right] \\
\frac{d C_{4}}{d t} & =\int d \lambda I^{*}(\lambda)\left[-\varepsilon_{4}(\lambda) C_{4} \phi_{42}+\varepsilon_{2}(\lambda) C_{2} \phi_{24}\right] \\
\frac{d C_{5}}{d t} & =\int d \lambda I^{*}(\lambda)\left[\varepsilon_{2}(\lambda) C_{2} \phi_{25}\right]
\end{aligned}
$$

where $C_{i}$ are the photoisomers concentrations, $\varepsilon_{i}(\lambda)$ the molar absorption coefficients and $\phi_{i j}$ the quantum yields of the photoreaction in the channel $i \Rightarrow j$. Photoisomers Provitamin D, Previtamin D, Tachysterol, Lumisterol and irreversible photoproducts Toxisterols are designated by the numbers 1, 2, 3, 4, 5 respectively.

$I^{*}(\lambda)$ is co-called photokinetics factor: $I^{*}(\lambda)=I_{0}(\lambda) \frac{1-10^{-A(\lambda)}}{A(\lambda)}$,

where $I_{0}(\lambda)$ is the intensity of the incident light at the wavelength $\lambda$, and $A(\lambda)$ is the solution absorbance:

$$
A(\lambda)=l \sum_{i=1}^{5} \varepsilon_{i}(\lambda) C_{i}
$$

The initial conditions for the concentrations are $C_{1}=100 \%$ and $C_{2}=C_{3}=C_{4}=C_{5}=0$.

The system of rate equations (2) can be numerically solved for any monochromatic or/and polychromatic light source with the intensity distribution $I_{0}(\lambda)$. To estimate the vitamin $\mathrm{D}$ synthetic capacity of sunlight at any latitude the solar spectra (either calculated using a radiative transfer model or measured by a spectroradiometer) might be used at the model input.

\section{Results and Discussion}

The two cuvettes of $0.5 \mathrm{~cm}$ thickness with 7 -DHC ethanol solution $(\mathrm{C}=20 \mathrm{mkg} / \mathrm{ml})$ were exposed at the roof of the Institute of Physics building in Kiev during 3 hours around noon (from 11:30 to 14:30 local time). Solar rays were kept normal to the cuvette surface by using a specially constructed mechanism for automatic tracking of the solar zenith angle. Further, the change of optical density at $\lambda=282 \mathrm{~nm}$ was determined for each measurement and concentrations of previtamin D were calculated using equation (1). The examples of the 7-DHC spectrum changes as a result of exposure to sunlight in summer and in autumn are shown in Figure 3.
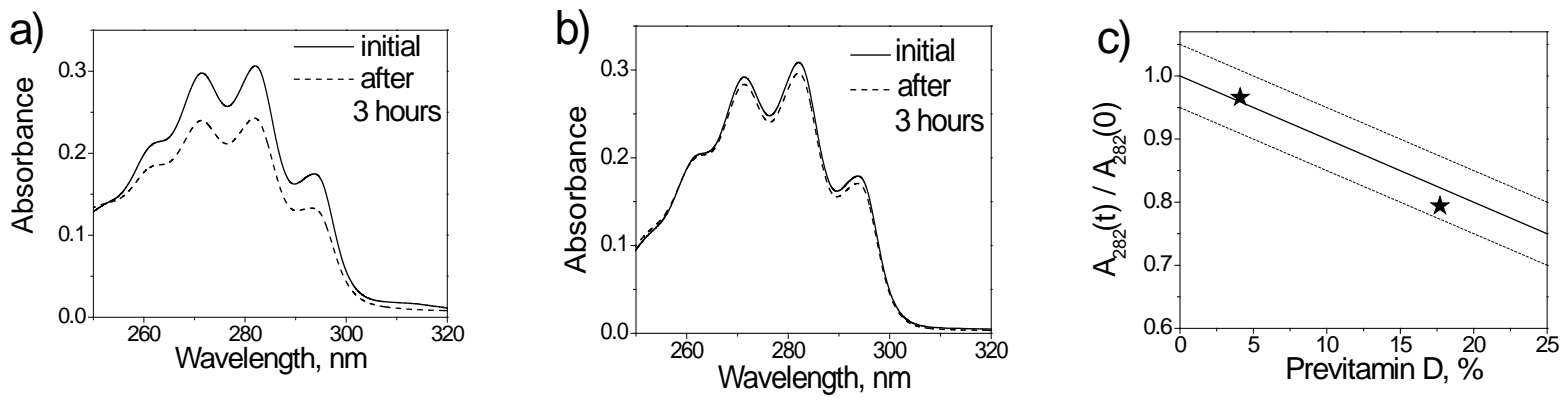

Figure 3. Absorption spectra of 7-DHC in ethanol before (solid line) and after (dash line) exposure to sunlight during 3 hours in early July (clear sky) (a) and September (scattered clouds) (b), and (c) concentrations of previtamin D calculated from the spectra using formula (1).

It is seen from Figure $3 \mathrm{c}$ that two previtamin D concentrations occur just within $\pm 90 \%$ prediction limit.

The examples of calculated kinetics of previtamin D accumulation using the system (2) and FASTRT program 
[8] for the three hours exposure to sunlight depending on the atmosphere properties (cloudiness and aerosol content) are shown in Figure 4.
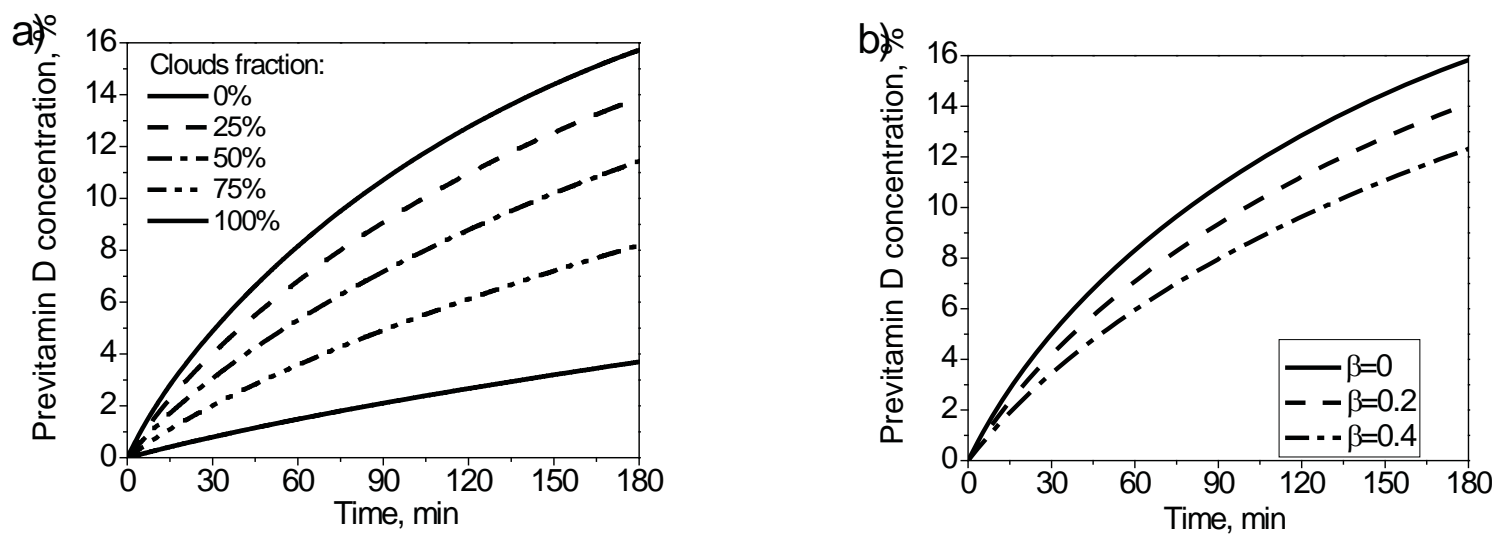

Figure 4. (a) Calculated kinetics of previtamin D photosynthesis in vitro on June 22 in Kiev (SZA $=27^{0} 52^{\prime}$, ozone 300 DU depending on cloudiness (cloud liquid water column $=400 \mathrm{gm}-2$ for clouds fractions $25-100 \%$, aerosol free sky, albedo 0 , exposure time $180 \mathrm{~min}$ ), (b) calculated effect of aerosol content on previtamin D photosynthesis in vitro $(\beta=$ aerosol optical thickness at $1 \mu \mathrm{m})[8]$.

It is apparent that effect of air pollution on previtamin D synthesis can be also estimated using the equations system (2) with solar spectra corrected for the absorption of gaseous air pollutants.

\section{Conclusion}

Initiation of endogenous synthesis of vitamin D in human skin is important biological function of solar UV-B radiation. Air pollution and the increase in tropospheric ozone hinder penetration of solar UVB to the Earth surface. Nowadays emerging research indicates that Vitamin D is more important to human health than previously thought. Vitamin D is recognized as a critical hormone that helps regulate the health of more than 30 different tissues, from the brain to the prostate [4].

Many ecologic, cohort and case control studies have shown that cancer mortality and in some cases cancer incidence depend inversely on ground level UV radiation. Prostate, colorectal, and breast cancers are most studied, although new data has shown that non-Hodgkin lymphoma [27, 28] and even survival with melanoma [20] are also inversely associated with sun exposure.

It is suggested that the major reason of a protective effect of sun exposure in the development of cancer and improved survival is just vitamin D synthesis which is a critical component of cellular networks that inhibit cellular proliferation and encourage apoptosis [30]. Therefore for validating the hypothesis many researchers today are focused on the measurements of sun exposure, serum vitamin D levels (and associated metabolites), and genetic variants that may affect vitamin D synthesis.

Although there are both beneficial and detrimental UV effects, it is an increase in the detrimental effects, associated with climate change, attract most attention. Concerns range from human health (increases in the incidence of skin cancer [31] to agriculture (reduction in crop yields), and to aquatic ecosystems (changes in the world's oceans) [32].

Recent findings on Europe's darker atmosphere in the solar UV-B [33] are in line with anticipated direct health consequences of climate change that include increases in air pollution-related illness. The studies in Canada and the United States indicated a high incidence of vitamin D deficiency in almost all populations, and chronic diseases associated with vitamin D deficiency are 25 to 50 percent more frequent in northern climates than among people living closer to equator $[5,34]$.

We think that global UV mapping as well as annual and daily forecasts should consider both erythemal and 'antirachitic' solar indices, and presented methods provide a means for direct monitoring of the vitamin D synthetic capacity of sunlight and the presented algorithm is useful for predictions of climate change effects on the vitamin D synthesis 


\section{References}

[1]. Ball, G.F.M. Vitamins: Blackwell Publishing Ltd., 2004; Chapter 8, pp188-233.

[2]. Mayer, A.C., Norman, A.W.; Vitamin D, Encyclopedia of Human Biology: Academic Press, NY, 1991; v.7, pp 859-871.

[3]. Holick, M. F., J. Cell. Biochem., 2003, 88, 296-307.

[4]. Norman, A. W.; Bouillon, R., Exp. Biol. Med., 2010, 235, 1034-1045.

[5]. Grant, W.B., Cancer 2002, 94, 1867-1875.

[6]. Holick, M.F., Biological Effects of Light: W. de Gruyter, Berlin, 1992; pp. 11-33.

[7]. Webb, A.R., Kline, L.W., Holick, M.F., J. Clin. Endocrinol. Metabol. 1988, 67, 373-378.

[8]. Engelsen, O., Kylling A.. Opt. Eng. 2005, 44, 041012-041018.

[9]. Hannelore Keller-Rudek, Geert K. Moortgat, MPI-Mainz-UV-VIS Spectral Atlas of Gaseous Molecules, www. atmosphere.mpg.de/spectral-atlas-mainz

[10]. McKinlay, A.F., Diffey, B.I., CIE J., 1987, 6, 17-22.

[11]. Terenetskaya, I., Proc. of the $2^{\text {nd }}$ CIE Expert Symposium "Lighting and Health", September 7-8, 2006, Ottawa, Canada, 2006; pp182-185.

[12]. Bouillon, R., Eisman, J., Garabedian, M., Holick, M., Kleinschmidt, J., Suda, T., Terenetskaya, I., Webb, A. CIE J. 2006, 174, 1-12.

[13]. Terenetskaya, I.P. SPIE Proc., 1994, 2134B, 135-140.

[14]. Galkin, O.N., Terenetskaya, I.P. J. Photochem. Photobiol. B: Biol. 1999, 53, 12-19.

[15]. Terenetskaya, I.P. Talanta 2000, 53, 195-203.

[16]. Terenetskaya, I. Agricult. Forest Meteorol. 2003, 120, 45-50.

[17]. Orlova, T. N., Terenetskaya, I. P. J. Appl. Spectr. 2009, 76, 240-244 (Transl. from Zhurnal Prikladnoi Spektroskopii, 2009, 76, 256-260).

[18]. Havinga, E. Experientia 1973, 29, 1181-1193.

[19]. Abillon E., Mermet-Bouvier R. J. Pharm. Sci. 1973, 62, 688-1691.

[20]. Jacobs, H.J.C., Havinga, E., Adv. Photochem. 1979, 11, 305-373.

[21]. Sternberg, J.C., Stillo, H.S., Schwendeman, R.H. Anal. Chem. 1960, 32, 84-90.

[22]. Davis, A, Deane, G.H.W., Diffey, B.L. Nature, 1976, 261, 169-170.

[23]. Terenetskaya, I.P., Orlova, T.M., Kirilenko, E.K., Eremenko, A.M., Galich, G.A., Ukrainian Patent \#93569, 25.02.2011, Priority date 24.03.2009.

[24]. Gvozdovskyy, I., Terenetskaya, I. In: Biologic Effects of Light: Academic Publishers, Boston, 2002, pp 341353.

[25]. Terenetskaya, I.P., Orlova, T.M. Proc. of SPIE 2008, 6991, 69911-F 1 - 7.

[26]. Orlova, T., Terenetskaya, I. Proc. of SPIE 2008, 7003, 70031O-1 - 8

[27]. Hughes, A. M., Armstrong, B. K., Vajdic, C. M , Turner, J., Grulich, A. E , Fritschi, L., Milliken, S., Kaldor, J., Benke, G., Kricker, A. Int J Cancer 2004, 112, 865-871.

[28]. Smedby K.E., Hjalgrim, H., Melbye, M., Torrang, A., Rostgaard, K , Munksgaard, L., Adami, J., Hansen, M., Porwit-MacDonald, A., Jensen, B. A, Roos, G., Pedersen, B. B., Sundstrom, C., Glimelius, B., Adami, H.O. J Natl Cancer Inst. 2005, 97 (3), 199-209.

[29]. Berwick, M., Armstrong, B. K, Ben-Porat, L., Fine, J., Kricker, A., Eberle C., Barnhill, R.L. J Natl Cancer Inst., 2005, 97 (3), 195-199.

[30]. Working Group of the Australian and New Zealand Bone and Mineral Soceity, Endocrine Society of Australia and Osteoporosis Australia. Med J Australia, 182 (6), 281-285 (2005).

[31]. Van der Leun, J.C., Piacentini, R.D.,de Gruijl, F.R. Photochem. Photobiol. Sci. 2008, 7, 730-733.

[32]. Cracknell, A.P., Varotsos, C. A. Environmental Science and Pollution Research 2007, 14, 384-387.

[33]. Seckmeyer, G., Glandorf, M., Wichers, C., McKenzie, R., Henriques, D., Carvalho, F., Webb, A., Siani, A-M., Bais, A., Kjeldstad, B., Brogniez, C., Werle, P., Koskela, T., Lakkala, K., Grobner, J., Slaper, H., denOuter, P. and Feister, U. Photochem. Photobiol. Sci. 2008, 7, 925-930.

[34]. Holick, M. F. Mayo Clin. Proc., 2006, 81, 353-373. 